\title{
Scale-dependent functions in statistical hydrodynamics: a functional analysis point of view
}

\author{
M.V.Altaisky \\ Joint Institute for Nuclear Research, Dubna, 141980, Russia \\ Space Research Institute, Profsoyuznaya 84/32, Moscow, \\ 117810, Russia
}

Revised: Aug 3, 1997

\begin{abstract}
Most of dynamic systems which exhibit chaotic behavior are also known to posses self-similarity and manifest strong fluctuations of all possible scales. The meaning of this terms is not always the same. In the present note we make an attempt to formulate the problem in the framework of functional analysis. The statistical hydrodynamics is taken as a vivid physical example.
\end{abstract}

\section{Introduction}

The problem of adequate mathematical description of hydrodynamic turbulence is one of the oldest but not yet solved problems in physics. The transition from laminar fluid flow to highly irregular chaotic regime was first discussed yet by Leonardo da Vinci. Later, when the Navier-Stokes equation (NSE)

$$
\partial_{t} \mathbf{v}+\mathbf{v} \cdot \nabla \mathbf{v}=-\nabla p+\nu \Delta \mathbf{v}, \quad \nabla \cdot \mathbf{v}=0
$$

which describes an incompressible fluid flow was written down, it was believed that (11) itself may contain all turbulence. It seems quite natural in the light 
of modern developments in dynamical chaos, that even systems with a few degrees of freedom sometimes show unpredictable chaotic behavior.

The turbulence problem is much more complicated. The velocity field $\mathbf{v}(\mathbf{x}, t)$ has $3 \times 3 D$ continuum degrees of freedom - thus we have a field theory problem. The dynamical object - the velocity field $\mathbf{v}(\mathbf{x}, t)$ itself is a square-integrable function defined on $I^{3} \times \mathbb{R}^{1}$ space.

The crucial step in turbulence theory was done by A. N. Kolmogorov in a number of short papers Kol41b, Kol41d, Kol41a, known as K41 theory. It was argued, that the turbulence, as a chaotic phenomenon, should be described in terms of random functions $\mathbf{v}(\mathbf{x}, t, \cdot)$ (The ideas of statistical description of turbulence have been already discussed by Taylor [Tay35]). It was suggested to consider the turbulence as a multi-scale phenomenon, formed of velocity fluctuations of all possible scales. The typical size $L$ of dominating fluctuations of average amplitude $U$ is related to the Reynolds number $R e=\frac{U L}{\nu}$, which determines the transition from laminar to turbulent flow. Following [Fri95] we present K41 hypothesis in the form

H1 In the limit $R e \rightarrow \infty$, all possible symmetries of the NSE, usually broken by the mechanisms reducing the turbulent flow, are restored in a statistical sense at small scales and away from boundaries.

H2 Under the same assumptions as in H1, the turbulent flow is self-similar at small scales, i.e.:

$$
\delta v(r, \lambda l) \stackrel{l a w}{=} \lambda^{h} \delta v(r, l)
$$

where $v(r, \lambda l)=v(r+\lambda l)-v(r)$. (The equality-in-law means the coincidence of all statistical momenta.)

H3 Under the same assumptions as in $\mathrm{H} 1$ and $\mathrm{H} 2$, the turbulent flow has a finite non-vanishing rate $\epsilon$ of dissipation of energy per unit of mass.

Using these three assumptions Kolmogorov has derived the two-thirds law

$$
\left\langle(\delta v(l))^{2}\right\rangle=C \epsilon^{2 / 3} l^{2 / 3},
$$

the basic empirical law of fully developed turbulence.

The scaling behavior (3) of velocity field $v$ means that we have to deal with functions of typical behavior

$$
|v(r+l)-v(r)| \sim l^{h}
$$


with $h \leq 1$, i.e. with non-differentiable functions; with nontrivial Hölder exponent $h=1 / 3$, if H3 hypothesis is strictly valid. The Hölder condition $|v(r+l)-v(r)| \leq l^{h}$ is not very rare in physical problems: it can be found in condensed matter physics, quantum field theory etc. - it becomes very significant only since the resolution $(l)$ itself becomes a physical parameter, and that is why we ought to consider some objects of the form $v(x, l, \cdot)$, which are not yet defined. This problem is actively discussed amongst the turbulence community, but up to the authors knowledge is beyond functional analysis research, except for the problems directly related to wavelet analysis [Hol94].

In present paper we investigate the problem of resolution dependent functions. The research was highly inspired by the fact, that there are at least two approaches to resolution dependent objects. First, based on the decomposition with respect to the group of affine transformations, is known as wavelet analysis. Second approach was exported from quantum field theory to turbulence and is based on the consideration of the Fourier transforms of functions cut at some large momentum $k=\Lambda_{\text {cut-off }}$.

An attempt to consider the problem as a whole and join these two approaches is presented below.

\section{Problem}

Self-similarity is a synonym of scale-invariance. To be scale-invariant means to have same properties at different scales. Classical fractals are scaleinvariant by construction. Brownian motion is self-similar: if we look at the trajectory of Brownian particle at different resolution of a microscope we will observe more or less the same picture.

As physical systems are considered, the word self-similarity is more frequently attributed to their dynamics than geometry.

The self-similarity of hydrodynamic velocity field fluctuations $\left\langle(\delta v(l))^{2}\right\rangle \sim$ $l^{2 / 3}$ is attributed to behavior of turbulent velocity field measured at different spatial scales. For hydrodynamical velocity field it is physically clear, that the measurement at scale $l_{0}$ necessarily implies averaging of molecular velocities over certain space domain of typical size $l_{0}$. This procedure can be generalized 
to "an averaging of a function up to scale l" [DG96]

$$
\phi_{l}(\mathbf{x})=l^{-D} \int_{|y|<l} \phi(\mathbf{x}-\mathbf{y}) d^{D} \mathbf{y}
$$

There are at least two conjectures here:

1. The existence of "true" (with no scale) field $\phi_{l}(\mathbf{x}): l \rightarrow 0$.

2. The homogeneity of the measure $d \mu(\mathbf{y})=d^{D} \mathbf{y}$.

Physically, it is quite clear, that two different fields $\phi_{l}(\mathbf{x})$ and $\phi_{l^{\prime}}(\mathbf{x})$ live in different functional spaces if $l \neq l^{\prime}$. It is meaningless, say, to subtract their values. Therefore, the velocity field of hydrodynamic turbulence is something more than a random vector field defined on $R^{D} \times R$.

To characterize the turbulent velocity at certain point $\mathbf{x}$ we ought to know the collection of velocity values $\left\{\phi_{l}(\mathbf{x})\right\}$ at a set of scales labeled by $l$. The set of scales may be countable

$$
l=l_{0}, k l_{0}, k^{2} l_{0}, k^{3} l_{0}, \ldots,
$$

say $k=1 / 2$ for period-doubling decomposition, or continuous.

To characterize this set it was proposed in [DG96 to use a collection of unit fields at different scales - a "reference field" $\left\{\mathcal{R}_{l}(x)\right\}$. The principal question arising here, is how to describe the interaction of the fluctuations of different scales. Practically, this problem is often coup with by decomposition of "real" (out-of- scale) field into slow (large-scale) and fast (small-scale) components.

$$
\phi=V+v, \quad \text { where } \quad \mathrm{M} v=0
$$

(M means the averaging, or mathematical expectation, here and after.) In this approach the slow component $V$ governs the equation for $v$ and the even-order moments of $v$ contribute to the equation for $V$.

From the other hand, as we know from both Kolmogorov's theory and RG approach, there are no absolute scales in hydrodynamics, except for dissipative scale and external scale (the size of the system). So, at least at this middle - the Kolmogorov's range - the equations should be scalecovariant. The structure which reveals here looks like a fiber bundle over $R^{D}$, with leaves labeled by scale. The fluctuations of different scales may be 
dependent or independent for various physical situations; but at least some similarity should be present.

To construct a basic system on this bundle let us follow the ideas of multi-resolution analysis Mal86. Let us construct a system of functional subspaces $\left\{V_{i}: V_{i} \subset \mathcal{H}\right\}$, Where $\mathcal{H}$ is a space of physical observables. Let the system $\left\{V_{i}\right\}$ be such, that

1.

$$
\ldots \subset V_{2} \subset V_{1} \subset V_{0} \subset \ldots
$$

2.

$$
\cap_{\infty} V_{i}=\emptyset, \quad \overline{\cup_{\infty} V_{i}}=\mathcal{H}
$$

3. Subspaces $V_{i}$ and $V_{i+1}$ are similar in some way.

$$
f(x) \in V_{j} \leftrightarrow f(k x) \in V_{j-1},
$$

$$
\text { if }\left\{\phi_{i}(x)\right\}_{i \in I} \text { forms basis in } V_{j} \text { then }\left\{\phi_{i}(k x)\right\}_{i \in I} \text { forms basis in } V_{j-1}
$$

If the sequence $\left\{V_{i}\right\}$ is bounded from above, the maximal subspace is called the highest resolution space. Let it be $V_{0}$. Then any function from $V_{1}$ can be represented as a linear span of $V_{0}$ basic vectors. Therefore, the basis $\phi_{0}$ of the highest resolution space provides a basis for a whole bundle.

It seems attractive to generalize MRA axioms to the case of continuous set of scales. Since the chain of subspaces described above implies sequential coarse graining of the finest resolution field, some details are being lost in course of this process. The lost details can be stored into the set of orthogonal complements

$$
V_{0}=V_{1} \oplus W_{1}, \quad V_{1}=V_{2} \oplus W_{2}, \ldots
$$

So, $\mathcal{H}=\overline{\sum_{k} W_{k}}, W_{k} \cap W_{j}=\emptyset$ if $j \neq k$, and the system $\left\{W_{i}\right\}$ can be considered instead of $\left\{V_{i}\right\}$. The former has the structure of $\sigma$-algebra, and thus it is suitable integration.

The fact, that velocities $\phi_{l}$ and $\phi_{l^{\prime}}$ live on different leaves suggest that their Fourier decomposition should be taken separately at their leaves

$$
\phi_{l}(x)=\int \exp \left(-\imath k^{(l)} x^{(l)}\right) \tilde{\phi}_{l}\left(k^{(l)}\right) d \mu_{L}^{(l)}\left(k^{(l)}\right),
$$


or some other care should be taken about it in order not to mix fluctuations with the same wave vectors but contributing to different scales. The choice of the left-invariant measure $d \mu_{L}^{(l)}\left(k^{(l)}\right)$ is restricted by the fact, that velocity components measured at certain scale are mainly concentrated close to this

scale. So the measure can be expressed as $d \mu_{L}^{(l)}(k)=d k W\left(\left|l^{-1}-a k\right|\right)$, where $W$ vanish at $x \rightarrow \pm \infty, a$ is a constant.

The decomposition (7) turns to be a kind of Gabor transformation Gab46]. The measure can $d \mu$ can be explicitly scale-dependent, since the probability spaces $\left(\Omega_{l}, \mathcal{U}_{l}, P_{l}\right)$ depend on scale..

At this point we arrive to the difference from standard wavelet approach, where the probability space is completely determined at finest resolution scale. However, if we accept the hypothesis that fluctuations of different sizes are statistically independent, we have to define the probability spaces separately.

\section{Wavelet realization}

We start our construction of multi-scale description with simplistic onedimensional case, which is however of practical importance since only one component of velocity field is often measured.

Any square integrable function $f(t) \in \mathrm{L}^{2}(\mathrm{R})$ can be represented as a decomposition with respect to the representations of affine group

$$
\begin{gathered}
t^{\prime}=a t+b \\
f(t)=C_{\psi}^{-1} \int \frac{1}{\sqrt{a}} \psi\left(\frac{t-b}{a}\right) W_{\psi}(a, b) \frac{d a d b}{a^{2}},
\end{gathered}
$$

which is just another form of the partition of unity with respect to representation of affine group acting on a Hilbert space [GJP85]

$$
\hat{1}=C_{\psi}^{-1} \int_{G} U^{*}(g)|\psi\rangle d \mu_{L}(g)\langle\psi| U(g),
$$

which holds if there exists such $\psi \in \mathcal{H}$, that

$$
C_{\psi}:=\frac{1}{\|\psi\|_{2}^{2}} \int_{G}|\langle\psi, \mid U(g) \psi\rangle|^{2} d \mu_{L}(g)<\infty
$$


holds; $d \mu_{L}(g)$ denotes left-invariant measure on $G$. The scalar products

$$
W_{\psi}(g) f:=\langle f, U(g) \psi\rangle
$$

are known as wavelet coefficients.

For the case of affine transformation group (8) the normalization constant $C_{\psi}$ can be easily evaluated in Fourier space

$$
C_{\psi}=\int_{-\infty}^{\infty} \frac{|\tilde{\psi}(k)|}{k} d k=2 \int_{0}^{\infty} \frac{|\tilde{\psi}(k)|}{k} d k,
$$

where $\psi(t)=\frac{1}{2 \pi} \int \exp (\imath k t) \tilde{\psi}(k) d k$. For the affine group (8)

$$
U(a, b) \psi(x):=\frac{1}{\sqrt{a}} \psi\left(\frac{x-b}{a}\right) ; \quad d \mu_{L}(a, b)=\frac{d a d b}{a^{2}}
$$

The corresponding wavelet coefficients are

$$
W_{\psi}(a, b)=\int \frac{1}{\sqrt{a}} \overline{\psi\left(\frac{t-b}{a}\right)} f(t) d t
$$

For practical analytical calculations it is often more efficient to calculate wavelet coefficients in Fourier representation, since multiplication should be done then instead of convolution:

$$
W_{\psi}(a, b) f=\frac{1}{2 \pi} \int \sqrt{a} \exp (\imath k b) \bar{\psi}(a k) \tilde{f}(k) d k
$$

and similar for reconstruction (9). The decomposition (12) and its inverse (9) are known as wavelet analysis (See e.g. Dau88 for general review.) The scalar product (12) is readily seen to be the projection of the original "no-scale" function $f$ to the subspace $W_{a}$ of MRA system 6 .

If $f$ is a random function defined on a probability space $(\Omega, \mathcal{A}, P)$, the wavelet coefficients

$$
W_{\psi}(a, b, \cdot)=\int \frac{1}{\sqrt{a}} \overline{\psi\left(\frac{t-b}{a}\right)} f(t, \cdot) d t
$$

are also random; the stochastic integration is implied. As it is known from the theory of stochastic processes, any random function $\xi(t, \omega), t \in \mathbb{R}^{1}, \omega \in \Omega$ can be represented in a spectral form

$$
\xi(t)=\int \phi(t, \lambda) \eta(d \lambda)
$$


where $\phi(t, \lambda)$ is a square-integrable function, $\eta(d \lambda)$ is a stochastic measure

$$
\mathrm{M} \eta(d \lambda)=0, \quad \mathrm{M}|\eta(d \lambda)|^{2}=F(d \lambda) .
$$

The particular form of the spectral representation (15) is Fourier representation

$$
\xi(t)=\int \exp (\imath \lambda t) \eta(d \lambda)
$$

In a multi-scale case we can introduce a collection of random processes, each of which belongs to its own leaf of MRA, labeled by resolution parameter $a$

$$
\xi_{a}(t)=\int \phi_{a}(t, \lambda) \eta_{a}(d \lambda) .
$$

The peculiarity of stochastic case is, that in contrast to the decomposition of a function with respect to given basic wavelet $\psi(t)$, the function $\phi_{a}(t, \lambda)$, which depends on both the properties of random process itself and filtering properties of measuring equipment, is not known exactly. Therefore, we have to construct a decomposition, which has a well defined limit to deterministic case and can be tackled without the exact specification of the form of basic wavelet $\psi$.

A straightforward way to do it, is to factorize the scaling part of the left-invariant measure from "purely stochastic part"

$$
\xi(t)=\int \phi_{a}(t, \lambda) \eta_{a}(d \lambda) \frac{d a}{a}
$$

or in the spectral form

$$
\xi(t)=\frac{1}{2 \pi C_{\psi}} \int e^{\imath \lambda t} \tilde{\psi}(\lambda a) \eta_{a}(d \lambda) \frac{d a}{a},
$$

where $\eta_{a}(d \lambda)$ can be considered as generalized wavelet coefficients, the existence of which does not require the existence of "no-scale" prototype.The left invariant measure $d \mu(a)=d a /(2 \pi a)$ on the multiplicative group $x^{\prime}=a x$ instead of (8), since translations are already incorporated into the exponent; $2 \pi$ multiplier is introduced for the convenience of Fourier transform.

The representation (16) was constructed only to meet the non-stochastic limit and is not unique. For instance, we can redefine the spectral measure to incorporate both the properties of the signal and that of measuring apparatus

$$
\xi(t)=\int e^{\imath \lambda t} \hat{\eta}_{a}(d \lambda) \frac{d a}{a} .
$$


The specific energy-per-scale density can be easily evaluated then

$$
\begin{aligned}
\int E(a) d a & =\mathrm{M} \int \xi(t) \bar{\xi}(t) d t \\
& =\mathrm{M} \int \exp \left(\imath t\left(\lambda_{1}-\lambda_{2}\right)\right) \eta_{a_{1}}\left(d \lambda_{1}\right) \bar{\eta}_{a_{2}}\left(d \lambda_{2}\right) d \mu\left(a_{1}\right) d \mu\left(a_{2}\right) d t \\
& =\mathrm{M} \int \eta_{a_{1}}(d \lambda) \bar{\eta}_{a_{2}}(d \lambda) d \mu(a) \\
& =\int F\left(a_{1}, a_{2} ; d \lambda\right) d \mu\left(a_{1}\right) d \mu\left(a_{2}\right)=E
\end{aligned}
$$

The equation (17) is a stochastic counterpart of a well known equation for wavelet energy per scale

$$
E(a)=C_{\psi}^{-1} \int \frac{|W(a, b)|^{2}}{a^{2}} d b=\frac{1}{2 \pi C_{\psi}} \int \frac{|\hat{W}(a, k)|^{2}}{a} d k
$$

Similar spectral characteristics have been already used for the analysis of turbulent data Ast96.

If random functions are considered the equation (18) can be rewritten in RG-like form

$$
\frac{\partial E}{\partial \ln a}=\frac{1}{2 \pi C_{\psi}} \mathrm{M} \int|\hat{W}(a, k)|^{2} d k
$$

where $\hat{W}(a, k)=\overline{\tilde{\psi}(a k)} \tilde{f}(k)$ can be understood as the original noisy signal $f$ perceived by filter $\psi$, i.e. as generalized wavelet coefficients, not necessary having "no-scale" prototypes, cf.eq.16.

The logarithmic derivative at the l. h. s. of (19) is exactly that of renormalization group equation. However, the r. h. s of this equation was obtained without any cutoff assumptions, it was formally derived from the decomposition of initial ("infinite resolution") signal filtered by window function $\psi$.

Physically, the existence of the infinite resolution limit is often meaningless. For instance, the behavior of electromagnetic coupling constant at Plank scale is just a nonsense. The same happens in hydrodynamics when the scale parameter comes close to the mean free path. However, there is a principal difference between hydrodynamical turbulence description and quantum field theory. The scale $l$ (resolution) becomes a physical measurable parameter in hydrodynamics, and thus $\hat{W}(a, k)$ can be considered as Fourier components of the velocity field fluctuations of different typical sizes. The study of the behavior of $\hat{W}(a, k)$ can provide more consistent picture of what happens at different scales than standard Fourier decomposition. 


\section{High frequency cut-off}

Being utmost scale-invariant at moderate scales, the behavior of turbulent velocity field changes when approaching the smallest and largest scales, between which the hydrodynamical description is valid. The former is the Kolmogorov dissipative scale $(\eta)$, the latter is the size of the system. The size of the system can often be set to infinity with no harm to physics; whilst the dissipative scale is of physical importance, since the energy dissipation rate $\bar{\epsilon}$ is very constant which determines the turbulence behavior in inertial range.

That is why in RG, as well as in spectral calculations, the cut-off dependent velocity field is often considered

$$
v_{F}^{<}(x)=\frac{1}{(2 \pi)^{d}} \int_{|k|<F} \exp (\imath k x) \tilde{v}(k) d k .
$$

The cumulative energy of all harmonics with wave vectors less or equal to the cut-off value $F$ is one of the main spectral characteristics of developed turbulence

$$
\mathcal{E}(F)=\frac{1}{2} \mathrm{M} \int \overline{v_{F}^{<}(x)} v_{F}^{<}(x) d^{d} x=\frac{1}{2} \mathrm{M} \int_{|k|<F} \overline{\tilde{v}(k)} \tilde{v}(k) \frac{d^{d} k}{(2 \pi)^{d}} .
$$

Similarly, we can consider the cumulative energy of all velocity fluctuations with typical size greater or equal to a given $A$. For simplicity let us consider a one-component velocity field considered as a function of time

$$
\begin{aligned}
E(A) & =\frac{1}{2} \mathrm{M} \int_{|a| \leq A} \overline{v(t)} v(t) d t=\frac{1}{C_{\psi}} \mathrm{M} \int_{a=A}^{\infty}\left|W_{\psi}(a, b) v\right|^{2} \frac{d a d b}{a^{2}} \\
& =C_{\psi}^{-1} \int_{A}^{\infty} \frac{|\tilde{\psi}(y)|^{2}}{y} d y \cdot \mathrm{M} \int|\tilde{v}(k)|^{2} \frac{d k}{2 \pi}
\end{aligned}
$$

where

$$
\lim _{A \rightarrow 0} 2 \int_{a=A}^{\infty} \frac{|\tilde{\psi}(y)|^{2}}{y} d y=C_{\psi}
$$

and

$$
E=\frac{1}{2} \mathrm{M} \int|\tilde{v}(k)|^{2} \frac{d k}{2 \pi}
$$

is the total energy of all velocity fluctuations. 
For non-vanishing $A$

$$
E(A)=F(A) E, \quad \text { where, } \quad F(A)=\frac{\int_{A}^{\infty} \frac{|\tilde{\psi}(y)|^{2}}{y} d y}{\int_{0}^{\infty} \frac{|\tilde{\psi}(y)|^{2}}{y} d y}
$$

For a better definiteness, let us calculate the filtering function $F(A)$ for a particular family of vanishing momenta wavelets

$$
\psi_{n}(x)=(-1)^{n} \frac{d^{n}}{d x^{n}} \exp \left(-x^{2} / 2\right), \quad \tilde{\psi}_{n}(k)=\sqrt{2 \pi}(-\imath k)^{n} \exp \left(-k^{2} / 2\right),
$$

often used for studying of hydrodynamical velocity field MBA91, MBA93.

The normalization constant for this family is

$$
C_{n}=2 \pi \int_{-\infty}^{\infty} k^{2 n-1} e^{-k^{2}} d x=2 \pi \Gamma(n)
$$

and so

$$
F_{n}(A)=\frac{\int_{A^{2}}^{\infty} y^{n-1} e^{-y} d y}{\Gamma(n)} .
$$

The derivative of cumulative energy with respect to logarithmic measure $d a / a$ is

$$
\frac{\partial E}{\partial \ln A}=E \frac{\partial F_{n}(E)}{\partial \ln A}=-\frac{\partial A^{2}}{\partial \ln A} f_{n}\left(A^{2}\right)
$$

where $f_{n}(x)=x^{n-1} e^{-x} / \Gamma(n)$. So we arrive at $\mathrm{RG}$ like equation

$$
\frac{\partial E}{\partial \ln A}=-\frac{2 A^{2 n} \exp \left(-A^{2}\right)}{\Gamma(n)} E
$$

For sufficiently small $A$ the exponential term is close to unity, and thus the behavior is approximately proportional to $A^{2}$.

\section{Self-similarity in a bounded domain of scales and Scale-relativity}

A direct approach to scale dependent functions, which was proposed by Nottale[Not93], is based on the assumption that physics is scale dependent, 
but scale covariant. The former means to give up the differentiability and consider functions $v(x, l)$ with $\lim _{l \rightarrow 0} \frac{\partial v(x, l)}{\partial x}$ not necessary existing; the latter imposes generalized scale covariant equation

$$
\frac{\partial v(x, l)}{\partial \ln l}=\beta(v(x, l)),
$$

which states that scale behavior of a scale-dependent function is completely determined by the value of this function at a given scale.

The benefit of scale relativity Not93 approach is to provide a possibility to account for the processes which admit self-similarity only at a limited domain of scales

$$
v(\lambda l)=\lambda^{h} v(l), \eta \ll l \ll \Lambda .
$$

For a homogeneous scale dependent function (mono-fractal) $v=v_{0}(\lambda / r)^{\delta}$ where $\delta$ is independent of scale $r$. To get the additive, rather than multiplicative form for two sequential scale transformations, it is convenient to express them in logarithmic form :

$$
\ln \frac{v\left(r^{\prime}\right)}{v_{0}}=\ln \frac{v(r)}{v_{0}}+\mathbb{W} \delta(r),
$$

where $\delta\left(r^{\prime}\right)=\delta(r)=\delta, \mathbb{V}=\ln \left(r / r^{\prime}\right)$. In real hydrodynamic turbulence the multi-fractal behavior is observed

$$
\delta=\delta(r)
$$

In the inertial range $\eta \ll r \ll \Lambda$ the exponent $\delta$ is practically a constant, but closer to the limiting scales $\eta$ and $\Lambda$ the dependence of scale becomes significant.

The logarithmic form (28) suggests a direct generalization on the multifractal case $\delta=\delta(r)$, which is like the generalization of Galilelian transform to Lorenzian transform:

$$
\begin{aligned}
& X^{\prime}=\Gamma(\mathbb{V})[X-\mathbb{V} T] \\
& T^{\prime}=\Gamma(\mathbb{V})[A(\mathbb{V}) X+B(\mathbb{V}) T]
\end{aligned}
$$

where $T=\ln \left(l / l_{0}\right), X(T)=\ln \mathrm{M}\left(v_{l} / v_{0}\right)$, see DG96 for details. 
The composition of two scale transformations of this type behaves like a composition of two Lorentzian boosts: when approaching the unpassible limit, then instead of "Galilelian"

$$
\delta(r)=\delta_{0}\left(1-\ln ^{2}(\Lambda / r) / \ln ^{2}(\Lambda / \eta)\right)^{-1 / 2}
$$

for the power behavior of velocity field. The transformation group (29) can be used to construct a wavelet decomposition at a limited domain of scales. This, however, is the subject of the next paper (in preparation).

\section{Conclusion}

In present paper we give a mathematical framework for the analysis of functions which depend on scale. Usually, the scale-dependent functions express the value of a certain physical quantity measured at a point $x$ by averaging over a box of size $l$ centered at $x$. Such functions are often used in hydrodynamics, geophysics, signal analysis. One of the most known ways of treating resolution-dependent functions is to identify the size of the box $(l)$ with the inverse wave number of Fourier transform $k^{-1}=l$. The higher wave numbers are then cutted off. Sometimes this procedure leads to confusion (many problems of field theory approach to hydrodynamic turbulence originate from this confusion).

In our approach, using the ideas of wavelet analysis, we keep wave vectors $(k)$ and scales $(a)$ separately. We derive a renormalization group like equations, which can be used to study the energy distribution between different scales. The results can be applied for the investigation of turbulence velocity experimental data, as well for further theoretical research.

$$
* * *
$$

The author is grateful to Prof. G.A.Ososkov for critical reading the manuscript. I'm also grateful and to Profs. V.V.Ivanov and I.V.Puzynin for stimulating interest to this research.

The work was supported in part by ESPRIT project 21042 . 


\section{References}

[Ast96] N.M. Astaf'eva. Wavelet analysis: basic theory and some applications. Uspekhi Fizicheskih Nauk, 166(11):1145-1170, 1996. In Russian.

[Dau88] I. Daubechies. Orthonormal bases of compactly supported wavelets. Comm. Pure. Apl. Math., 41:909-996, 1988.

[DG96] B. Dubrulle and F. Graner. Possible statistics of scale invariant systems. J. de Physique II France, 6(5):797-815, 1996.

[Fri95] U. Frish. Turbulence. Cambridge University Press, 1995.

[Gab46] D. Gabor. Theory of communication. Proc. IEE, 93:429-457, 1946.

[GJP85] A. Grossmann, Morlet J., and T. Paul. Transforms associated to square integrable group representations. i. general results. J. Math. Phys., 26:2473-2479, 1985.

[Hol94] M. Holshneider. Functional calculus using wavelet transform. J. Math. Phys, 35(7):3745-3752, 1994.

[Kol41a] A.N. Kolmogorov. Dissipation of energy in locally isotropic turbulence. Dokl. Akad. Nauk SSSR, 32:16-18, 1941. Reprinted in Proc. R. Soc. Lond. A434, 15-17,(1991).

[Kol41b] A.N. Kolmogorov. The local structure of the turbulence in incompressible viscous fluid for very large reynolds numbers. Dokl. Akad. Nauk SSSR, 30:9-13, 1941. Reprinted in Proc. R. Soc. Lond. A434, 9-13,(1991).

[Kol41c] A.N. Kolmogorov. On degeneration of isotropic turbulence in an incompressible viscous liquid. Dokl. Akad. Nauk SSSR, 31:538-540, 1941.

[Mal86] S. Mallat. A theory for multiresolution signal decomposition: wavelet transform. Preprint GRASP Lab. Dept. of Computer an Information Science, Univ. of Pensilvania, 1986. 
[MBA91] J.F. Muzy, E. Bacry, and A. Arneodo. Wavelets and multifractal formalism for singular signals: Application to turbulence data. Phys. Rev. Lett., 67:3515, 1991.

[MBA93] J.F. Muzy, E. Bacry, and A. Arneodo. Multifractal formalism for fractal signals: The structure-function approach versus the wavelet-transform modulus-maxima method. Phys. Rev. E, 47:875-84, 1993.

[Not93] Laurent Nottale. Fractal Space-Time and microphysics: Towards a Theory of scale relativity. World Scientific, Singapore, 1993.

[Tay35] G.I. Taylog. Statistical theory of turbulence. Proc. R. Soc. Lond., 151:421-478, 1935. 